\title{
Control Software Design of Plant Microscopic Ion Flow Detection Motion Device
}

\author{
Lulu He ${ }^{1,2}$, Fubin Jiang ${ }^{2}$, Dazhou Zhu ${ }^{3}$, Peichen $\mathrm{Hou}^{2}$, Baozhu Yang ${ }^{4}$, Cheng Wang ${ }^{2}$, \\ and Jiuwen Zhang ${ }^{1}$ \\ ${ }^{1}$ Information Science \& Engineering, Lanzhou University, Lanzhou 730000, China \\ ${ }^{2}$ Beijing Research Center for Information Technology in Agriculture, Beijing 100097, China \\ ${ }^{3}$ Beijing Research Center of Intelligent Equipment for Agriculture, Beijing 100097, China \\ ${ }^{4}$ Beijing PAIDE Science and Technology Development Co., Ltd., Beijing 100097, China \\ lulu_purple@163.com, jiangfubin0702@126.com, \\ \{zhudz, houpc, yangbz, wangc\}@nercita.org.cn, zhangjw@lzu.edu.cn
}

\begin{abstract}
To realize in vivo, real-time, non-invasive and in-situ detection analysis on the dynamic changes of ion flows in plant tissues, organs, cells and others under natural conditions, this paper designed three-dimensional electronic control motion platform of high-accuracy so as to realize the highlyaccurate motion control in micron order and enable glass microelectrode to measure the plant tissues, organs and cells in its best state. The apparatus requires the portable design, so this paper chose the ion flow detection device with small size and high accuracy and micro integrative drive control module. Also graphical programming software LabVIEW was utilized to send serial port commands so as to realize the accurate and reciprocating motion of glass microelectrode at different positions and further accurately detect the plant microscopic dynamic ion flow parameter. The test showed that this control software could realize highly-accurate positioning of $0.6371 \mu \mathrm{m} / \mathrm{step}$ in threedimensional space and the software system could operate steadily, which provides strong supports for the plant microscopic ion flow detection.
\end{abstract}

Keywords: ion flow, LabVIEW, software control, accurate positioning.

\section{Introduction}

Same as the principles of non-invasive micro-test technique, plant microscopic ion flow detection technique is a new technique used to measure and study ion/ molecular flow. NMT (Non-invasive Micro-test Technique) is a general term of a wide mass of microelectrode techniques, including non-invasive scanning ion-selective electrode technique (SIET), which can obtain the ion or molecular flow inside and outside membrane of samples in premise of no destroy of test samples [1-2]. With profound three-dimensional measurement mode [3-4], this technique not only can measure the absolute concentration of ion/ molecular in stationary state but also can measure the motion rate and motion direction of plant samples. It has been widely used in the studies of plant cells and animal cells and also integrated with other microscopical techniques [5]. Some existing biological precise instruments have been used in the 
plant ion flow detection by experts at home and abroad, for example, Non-invasive Micro-test System by USA Younger Company. Through the automatic control of computer and precision machinery and on the basis of ion flow velocity calculation principle in Fick law, this paper carried out three-dimensional, real-time and dynamic measurement under the condition of no contacting with test samples to obtain the information about ion/ molecular concentration, flow velocity and motion direction of samples. However, in consideration of the expensive cost, complex structure and wires, large size and immovability, this apparatus cannot be applied in the plant ion flow detection under natural conditions.

On the basis of the small-size ion flow detection motion device and hardware basis of micro integrative stepper motor drive control module, this design carried out virtual instrument development and design according to the concrete demands of ion flow data collection and motion mode. LabVIEW control software was utilized to send serial port commands to control stepper motor, which drives ion flow detection device to control the microelectrode. In this way, the accurate positioning in $\mathrm{X}, \mathrm{Y}$ and $\mathrm{Z}$ directions can be realized and the gradient changes of ion concentration at different measurement points can be acquired. Furthermore, the absorption and diffusion rate and trend of certain target ions of the plant can be reflected. In this way, people can find the laws and characteristics of plant dynamic growth.

\section{Control System Constitution of Motion Device}

To satisfy the detection requirements of filed plant in-vivo ion flow, the apparatus shall be portable and have high accuracy and stability. To meet the above requirements, the designed control system in this paper is composed of four parts, upper computer, stepper motor drive control module, ion flow detection motion device and bundled control software of upper computer. Stepper motor drive control module consists of stepper motor drive control driver UIM242, control protocol converter UIM2501 and attached devices, which can connect multiple control drivers and realize the synchronous control of stepper motor by aid of its high integration level and micro and portable features. The ion flow detection motion device is respectively equipped with stepper motors in $\mathrm{X}, \mathrm{Y}$ and $\mathrm{Z}$ directions, which converts the input pulses into corresponding angular displacements. Then through elaborate mechanical drive, they are converted into executive components of linear displacement and their input variables are impulse sequences and the output results are the corresponding angles or linear increments, whose rotations are operated in settled angle step by step [6-7]. The accuracy of this three-dimensional motion device can reach $0.094 \mu \mathrm{m} / \mathrm{step}$, which can accurately positioning the sampling probe of ion flow detection motion device and control it to complete reciprocating motion. The bundled control software of upper computer owns sound human-computer interaction interface and good stability and portability.

\section{Overall Design of Control System Software}

This software system adopted LabVIEW virtual instrument development platform. The virtual instrument is the hardware platform which takes PC computer as the core and can be designed and defined by users. It has virtual panel and its core is software 
katechnology [8]. Because LabVIEW software can provide many controls whose appearances are similar to traditional instruments, it is convenient to create user interface. Users can define and manufacture various kinds of instruments according to their own demands, so this design adopted LabVIEW development platform. Function library of graphical programming software LabVIEW includes data acquisition, GPIB, serial port control, data analysis, data display and storage. The programming of LabVIEW software control mode is so easy that users can construct the required instrument system. Furthermore, sound human-computer interaction interface makes the control more convenient and flexible [9-11].

Visual graphical programming language covers front panel and program chart. Front panel, similar to the operation panel of the instrument, includes knob, on/off keys and others. Program chart is the core of the software, which can be used to realize the functions of control software. The program execution sequence depends on the data flow direction [12].

The software is based on LabVIEW human-computer interaction interface, which can send control commands on stepper motor control driver to control instruments through interaction interface and obtain the equipment operational condition. To execute electrode three-dimensional motion control and data acquisition operation, the control software is composed of four parts, serial port command packaging, RS232 serial port protocol transmission, information feedback and control software interface program. Serial port command packaging packages hexadecimal character string command into modules that can be called conveniently. RS232 serial port protocol transmission can connect the command and instrument and send the data to the stepper motor drive control driver according to its specified communication mode. After receiving the control command, the stepper motor drive control driver will analyze the commands, complete the specified motions and return to hexadecimal character string. Information feedback module is specific to analyze the returned value character string and timely reflect the motion state of stepper motor. At the same time the limit sensors in each direction of the three-dimensional motion device analyze the feedback information to get the level fluctuation function of sensors and achieve motion protection. Control software interface program can provide the interaction interface which controls corresponding motions of ion flow detection motion device. Through this interface, users can complete all the operations of ion flow detection.

\subsection{RS232 Serial Port Communication and Information Feedback}

PC serial port has point-to-point communication with ion flow detection motion device and all commands are based on 7-digit standard ASCII commands of RS232 Protocol. Protocol converter control the subordinate control driver with high speed through CAN Protocol. In this way, serial port communication mode is adopted. The correctness of commands is related to the accuracy of experimental data acquisition. Wrong command may result in the damage of sampling probe of ion flow. To avoid this, information feedback module was designed in LabVIEW control software, which made it convenient for users to see the sent commands and analyze the feedback 
information. In this way, the motion protection of ion flow detection motion device can be achieved.

LabVIEW provides powerful Virtual Instrument Software Architecture (VISA) library, which includes basic serial port operation functions such as serial port settings, serial port writing, serial port reading, serial port closing and so on [13]. To realize the serial port communication function, the serial port has been set as follows through serial port configuration functions. Baud rate $=9600$; each byte includes one start bit, 8-bit data bits and one stop bit. There is no flow control and parity check bit. If time-out is over $10 \mathrm{~s}$, waiting time of $50 \mathrm{~ms}$ is initiated. The serial port parameter configuration of both communication parties shall be same, or it will fail to work. Send the serial port commands in form of hexadecimal character string to the specific equipment of VISA resource name.

Wait for $50 \mathrm{~ms}$ delay before reading operation in order to determine the number of bytes in the receive buffer. Attribute nodes of Byte at Port return to the existing number of bytes in input buffer in order to guarantee the integrity of the received character string. Make use of reading function to read the data in the specific equipment of VISA resource name and the bytes of specific amount in the specific equipment. Then display the read data in the buffer as feedback information.

Feedback information is the information sent by control driver to upper computer. After receiving the command, the control driver will immediately send the confirmation message. If the command is wrong, it will not be executed. Once the required number of bytes actually read from the input buffer is finished or there is time-out error, this reading operation can be over.

\subsection{Accurate Positioning of Ion Flow Detection Motion Device by LabVIEW Control}

Plant microscopic ion flow detection experiment requires the three-dimensional microspur motion of microelectrode, which can enable the ion flow detection microelectrode to detect the ion absorption and release physiological properties of plant cells in optimum state. Therefore, the accurate positioning of ion flow detection motion device plays a vital role. This design adopted command-packaging motion control mode to respectively control the ion flow detection motion device in $\mathrm{X}, \mathrm{Y}$ and $\mathrm{Z}$ directions so as to realize the highly-accurate positioning of ion flow detection motion device and support the relevant data collection of ion flow. Take motions in $\mathrm{X}$ axis as an example and its accurate positioning chart is shown as Fig. 1. The motions in $\mathrm{Y}$ axis and $\mathrm{Z}$ axis are similar to that in $\mathrm{X}$ axis.

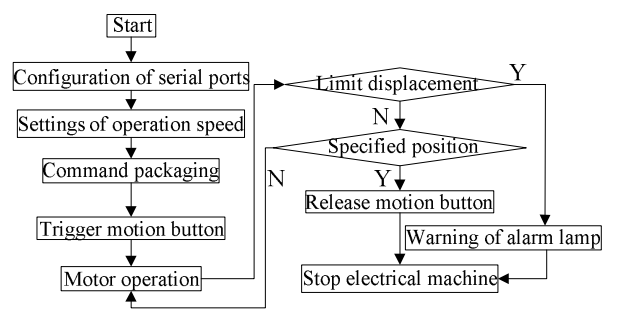

Fig. 1. Accurate positioning chart of stepper motor 
First, the address of stepper motor control driver which receives commands shall be assigned. Commands include operation speed, enabling and stop. "+" in the operation speed command represents forward motion while "-" represents backward motion. The motion control button adopted the trigger mode of maintaining conversion until release. Selection function was used, so once the motion control was triggered, the motion enabling command could be triggered. After being released, execute the stop command "OFF" and realize the accurate positioning of the probe of ion flow detection motion device. Its real-time performance was good. After the serial port communication was over, device dialog handle must be closed and the serial port resources shall be released, or there would occur errors in serial port resource when the program is operates next time. The forward motion control program chart in $\mathrm{X}$ axis is shown as Fig. 2.

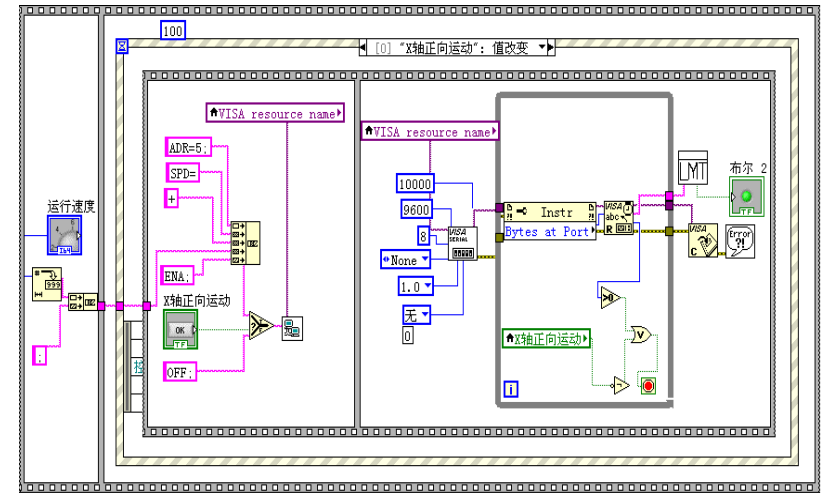

Fig. 2. Forward motion control program chart in $\mathrm{X}$ axis

\subsubsection{Motion Control and Command Confirmation}

Trigger forward motion button in $\mathrm{X}$ axis in the front panel and through serial port communication and information feedback subprogram, the confirmation information of the command can be received. Then determine whether the sent command is consistent with the user demands.

The message length is flexible and the maximum shall be 13 bytes. The feedback information is composed of the following parts, header, driver identification code, information identification code, information data and end mark.

1) Header represents the start of feedback information.

2) Driver identification code represents the site identification number of the control driver and the identification number of teach control driver is unique.

3) Information identification code indicates the attribute of the message.

4) Information data: control driver can immediately reply all the parameter information including the latest set one, read the feedback information and obtain the required parameter values through displacement.

5) End mark: the control driver adopts $0 \mathrm{xFF}$ as end mark to indicate the end of one message.

Input command is $\mathrm{ADR}=5$; $\mathrm{SPD}=6000$; $\mathrm{ENA}$; the aim is to enable $\mathrm{X}$ axis of control driver with identification number 5 to do enabling motion with speed of 6,000 
pulses per second. After receiving the command, the control driver immediately displays the confirmation information, AA05 D0FF AA05 B500 2E70 FFAA 057F 0400 2E70 00000000 00FF. The meaning of the feedback information is as follows.

AA: command confirmation feedback.

05: specify the command object as the control driver with identification number 5 .

D0: information identification code of driver site.

FF: end mark.

B5: information identification code of expected speed.

00 2E 70: rank according to 8-bit data byte, with high-order ahead and low-order behind, shown as Figure 3. Convert 7-bit data byte into 16-bit data through displacement and decimal system of 6,000. Namely the expected speed is 6,000 pulses per second, which is consistent with the user demands.

\begin{tabular}{|c|c|c|c|c|c|c|c|c|c|c|c|c|c|c|c|c|c|c|c|c|c|c|c|c|c|}
\hline \multicolumn{4}{|c|}{ Bit } & 1 & 5 & 14 & 13 & 12 & 11 & 1 & & 9 & 8 & & 7 & 6 & 5 & & 4 & 3 & 2 & & 1 & 0 & $\begin{array}{l}\text { decimal } \\
\text { svstem }\end{array}$ \\
\hline \multicolumn{5}{|c|}{ 16-bit data (binary system) } & & 0 & 0 & 1 & 0 & 1 & & 1 & 1 & & 0 & 1 & 1 & & 1 & 0 & 0 & & 0 & 0 & 6000 \\
\hline \multicolumn{7}{|c|}{ First byte $(00)$} & bit & \multicolumn{5}{|c|}{ Second byte (2E) } & bit & & & \multicolumn{5}{|c|}{ Third byte (70) } & bit & & & & \\
\hline Bit 7 & 6 & 5 & 4 & 3 & 2 & 1 & 0 & 7 & 6 & 5 & 4 & 3 & 2 & 1 & 0 & & 7 & 6 & 5 & 4 & 3 & 2 & 1 & 0 & \\
\hline Data 0 & 0 & 0 & 0 & 0 & 0 & 0 & 0 & 0 & 0 & 1 & 0 & 1 & 1 & 1 & 0 & & 0 & 1 & 1 & 1 & 0 & 0 & 0 & 0 & \\
\hline
\end{tabular}

Fig. 3. Conversion of data byte

7F: synthetic byte, whose format is shown as table 1 . The binary system of 7F is 01111111, which is one-to-one correspondence to the table. It can be converted that the stepper motor is forward enabling in form of 16-subdivision and automatically cut down the current when the motor stops.

Table 1. Synthetic byte in the feedback information

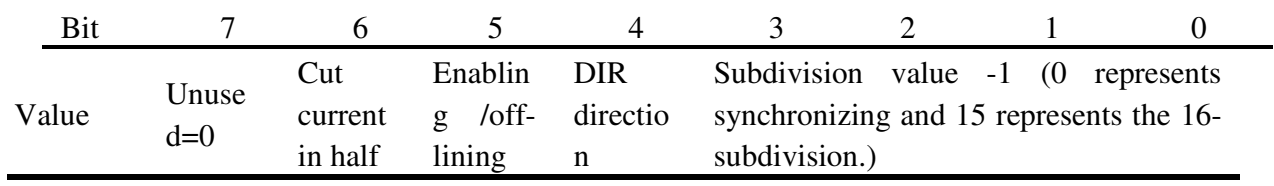

04: Current byte. Current value $=$ actual current $* 10$. If Current value is 4 , the actual current is $0.4 \mathrm{~A}$. The actually set current is consistent with this.

00 2E 70: the expected speed is 6,000.

00000000 00: the expected displacement is not set. Convert 5 data bytes into 32bit data to express the expected displacement. The conversion mode is similar to that of expected speed and here is not repeated.

\subsubsection{Motion Protection}

Ion flow detection motion device is precise instrument. The three dimensional motion displacement is expressed as $\mathrm{X}$ (slanted toward) $=\mathrm{Y}=\mathrm{Z} \geq 26 \mathrm{~mm}$ so as to protect the ion 
flow detection motion device from damage due to exceeding the limited range. The stepper motor is equipped with limit sensors respectively at the forward and backward motions in $\mathrm{X}, \mathrm{Y}$ and $\mathrm{Z}$ directions. How to determine whether the location of the stepper motor triggers the limit switch and after that how to control its motion state are the key techniques of motion protection of ion flow detection motion device. Therefore, this paper designed the motion protection subprogram and the limit program chart is shown as Fig. 4.

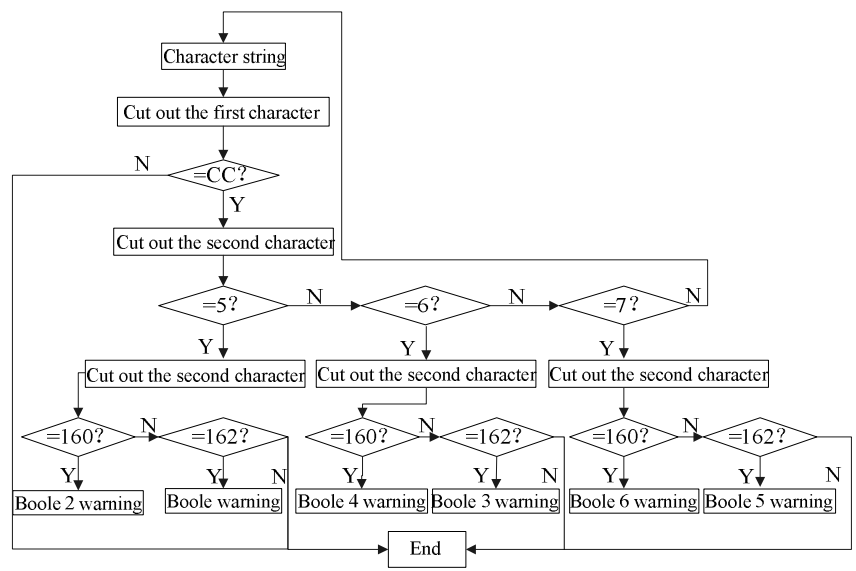

Fig. 4. Limit program chart

The limit sensor at both ends of ion flow detection motion device is connected with the sensor of stepper motor control driver. In this way, the event of sensor triggering occurs. The change notification of real-time state can response to the sensor event. There is feedback information in the reading buffer, which is still expressed in form of hexadecimal character string. Cut-out character string function is adopted to determine each byte. To prevent from the accident due to exceeding the limited displacement, once the limit sensor is triggered, immediately order the stepper motor to stop. Making use of the sensor event in the stepper motor control driver, when the limit sensor is at low level, the stepper motor stop working and the alarm lamp is lightened for warning.

The meanings of bytes are as follows.

(1) The first byte corresponds to CC, expressing the state feedback information.

(2) The second byte corresponds to one of 5, 6, or 7, namely the control driver identification codes respectively corresponding to $\mathrm{X}, \mathrm{Y}$ and $\mathrm{Z}$ directions of ion flow detection motion device.

(3) The third byte corresponds to A0 (whose decimal system is 160) and A2 (whose decimal system is 162), respectively representing that limit sensors of S1 and $\mathrm{S} 2$ are in the falling edge.

In case of forward motion of $\mathrm{X}$ axis, the feedback information displays character strings of four-byte, $\mathrm{CC} 05$ A0FF. The bytes can be converted that sensor S1 of control driver with site number of 5 is at low level, indicating that there is warning at 
the forward motion of $\mathrm{X}$ axis and the corresponding Boole warning lamp is triggered. Finally, the stepper motor stops motion. The warning of the alarm lamp in the front panel of the main program is shown as Fig. 5. Two alarm lamps respectively set in X, $\mathrm{Y}$ and $\mathrm{Z}$ directions represent the forward limit and backward limit. In this way, the motion protection of ion flow detection device can be achieved.

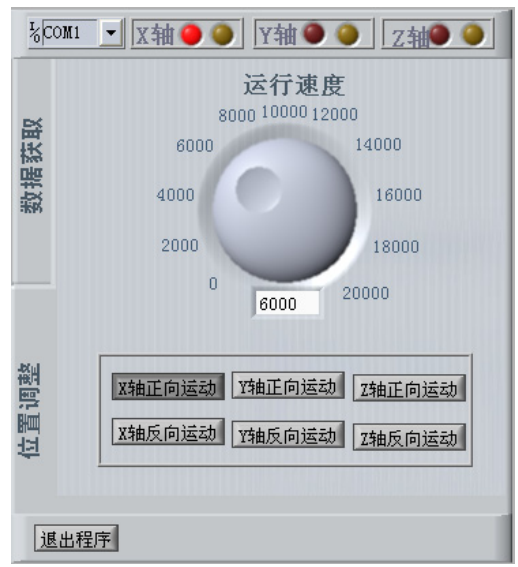

Fig. 5. Front panel of limit warning

\subsection{Reciprocating Motion of the Probe of Ion Flow Signals Controlled by LabVIEW}

After the accurate positioning of the probe of ion flow detection motion device, in consideration of the practical application demands of plant ion flow detection experiment, this paper collected reciprocating motion of the probe through the control signals of ion flow detection motion device. During the process of reciprocating motion, the required experiment data by users were further studied and explored. The program chart of the reciprocating motion module is shown as Fig. 6.

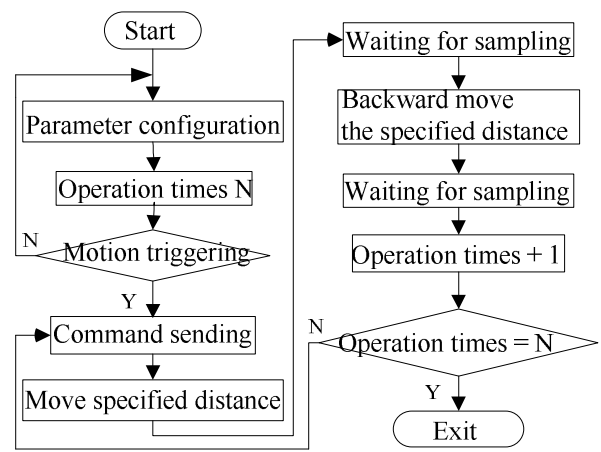

Fig. 6. Reciprocating motion chart 
The front panel of reciprocating motion is shown as Fig. 7. Its function is to order the ion flow detection motion device to do the reciprocating motion from origin point to specified position and back the origin point again according to the set operation times in $\mathrm{X}$ axis. After setting the operation speed, operation times, waiting time of sampling, expected displacement increment and other parameters, trigger the start motion and the stepper motor starts to move. After it reaches the expected position, pause the waiting time of sampling and return to the origin point. In this way, reciprocating motion takes shape.

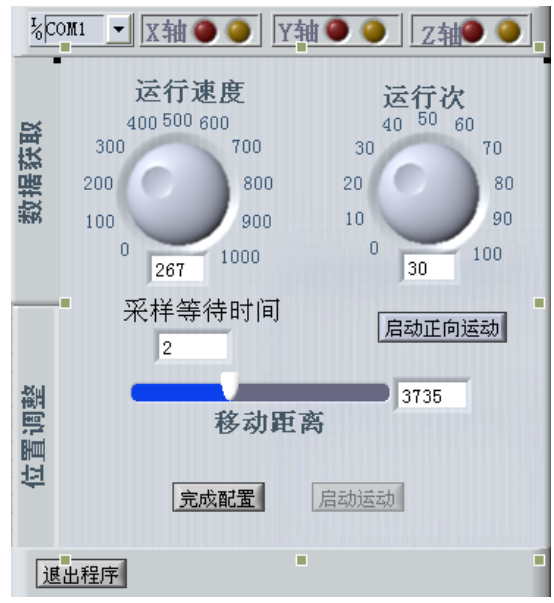

Fig. 7. Front panel of reciprocating motion

The sampling probe triggers forward motion and backward motion according to the set speed and displacement and after reaching the specified position, it waits for the sampling time. By setting the expected position as 0 and enabling command, the probe of ion flow sampling returns to the origin point again and completes one reciprocating motion. After the waiting time of sampling, which is same as that in the above procedure, the next reciprocating motion starts. Execute while loop for once and the operation times are taken as the cycle count of while loop. Until the set operation times set by users are completed and while loop is over, can it stops motion. Waiting time shall be calculated in the procedure. The motion time of stepper motor $=$ displacement distance/operation speed. Procedure waiting time $=$ operation time + sampling waiting time. The unit of operation time and sampling waiting time is second while that of procedure waiting time is millisecond. The conversion between these two units shall be carried out. Sampling waiting time can be set differently according to the experimental requirements of users.

Electric pulse signal received by stepper motor is converted into angle displacement or linear displacement. Through controlling the number of pulses, the angle displacement is controlled. In the plant microscopic ion flow detection experiment, ion flow detection motion device converts the motor power into linear motion. The reciprocating motion needs the microelectrode to accurately move to the 
specified position and the length is of micron order. By aid of amplification factor $\mathrm{k}$, angle displacement is converted into accurate distance length $\mathrm{S}$. Because the eyepiece of microscope has a scale with $10 \mu \mathrm{m} /$ square, people can test the precision through the relationship between pulses and real distance. The test results are showed as table 2 .

Table 2. The datas of measuring the accuracy

\begin{tabular}{cccccc}
\hline $\begin{array}{c}\text { Pulses } \\
(\text { number })\end{array}$ & $\begin{array}{c}\text { Real } \\
\text { distance 1 } \\
(\mu \mathrm{m})\end{array}$ & $\begin{array}{c}\text { Real } \\
\text { distance 2 } \\
(\mu \mathrm{m})\end{array}$ & $\begin{array}{c}\text { Real } \\
\text { distance 3 } \\
(\mu \mathrm{m})\end{array}$ & $\begin{array}{c}\text { Real } \\
\text { distance } 4 \\
(\mu \mathrm{m})\end{array}$ & $\begin{array}{c}\text { Mean } \\
\text { accuracy } \\
(\mu \mathrm{m} / \mathrm{step})\end{array}$ \\
\hline 10000 & 390 & 385 & 387 & 390 & 0.6208 \\
5000 & 198 & 199 & 200 & 199 & 0.6368 \\
2500 & 104 & 102 & 101 & 101 & 0.6538 \\
\hline
\end{tabular}

STP represented the relative displacement increment, defined with pulse count. In stepper motor control driver, 16 subdivision was executed, this experiment measured the stepper motor accuracy of $0.6371 \mu \mathrm{m} / \mathrm{step}$, so $0.6371 / 16^{*} \mathrm{STP}=\mathrm{S}, \mathrm{STP}=\mathrm{k} * \mathrm{~S}$ was utilized. In this way, people can deduce that $0.6371 / 16 * k^{*} \mathrm{~S}=\mathrm{S}$. So $\mathrm{k}=25$. The microscope imaging is showed as Fig. 8.

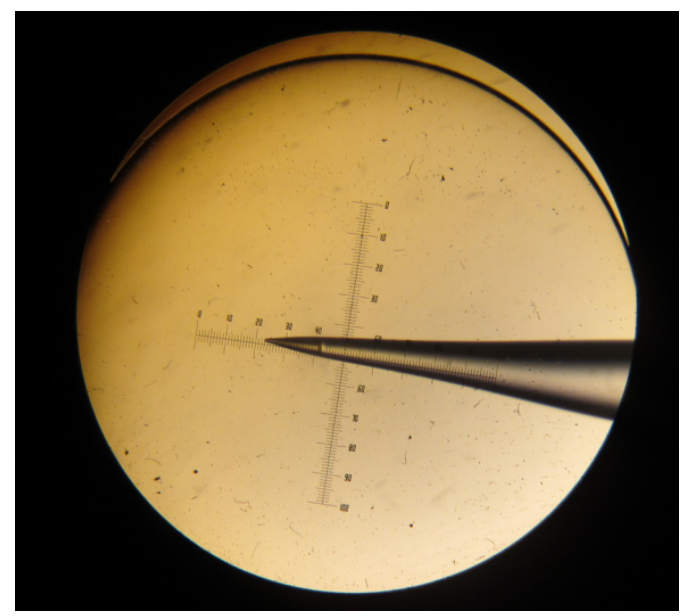

Fig. 8. The microscope imaging

\section{Functional Test on the Control Software of Ion Flow Detection Motion Device}

After set up the whole system, the functional test was carried out on the control software. The plant microscopic ion flow detection belongs to micro measurement, so 
naked eyes cannot observe the displacement distance of probe microelectrode. Therefore, photoelectric microscope was adopted. Dividing rule is equipped on objective lens, with $10 \mu \mathrm{m}$ division value. Move the probe into the view distance and people could clearly observe the movement of the probe. Via multi-measurement average, we got the accuracy was $0.6371 \mu \mathrm{m} / \mathrm{step}$. However, it is inevitable to generate errors. The reasons for errors are as follows. 1. The division value of photoelectric microscope is $10 \mu \mathrm{m}$, so the reading number in the measurement all were estimated numbers. 2. There existed accumulated errors during the mechanical rotation process but the accuracy of $0.6371 \mu \mathrm{m} / \mathrm{step}$ still could satisfy the $2 \mu \mathrm{m} / \mathrm{step}$ experimental requirement of plant ion flow detection. Also the motion device based on control software LabVIEW has sound real-time performance and stable performance.

\section{Conclusions}

By aid of LabVIEW development platform, the control on the motion device of plant microscopic ion flow detection is realized. The processing speed is fast and the realtime communication between GPIB and PC [14] can immediately feedback the motion information and have high accuracy and good real-time performance [15]. Three-dimensional microspur motion device based on this technique can satisfy the high requirements of precision measuring instrument and the control software can change control modes according to user demands at any time. The system can operate stably and have strong portability as well as accurate, flexible and portable features. However, the aesthetics of control software still need improvements. Repeated debugging and experimental detection as well as feedback of relevant experimenters are necessary for the improvement of the whole system. In this way, the usability of the software and the stability of the control software of plant microscopic ion flow detection motion device can be further improved and perfected.

Acknowledgment. Funds for this research was provided by the National key scientific instrument and equipment development project (2011YQ080052), Major national science and technology achievement transformation projects (finance construction [2012] No. 258) and Agricultural science and Technology Achievements Transformation Fund Project (2012GB2A00001).

\section{References}

1. Kunkel, J.G., Cordeiro, S., Yue, X., et al.: The use of non-invasive ion-selective microelectrode techniques for the study of plant development. In: Volkov, A.G. (ed.) Plant Electrophysiology-Theory and Methods, ch. V, pp. 109-137. Springer, Heidelberg (2005)

2. Xu, Y., Sun, T., Yin, L.: Application of non-invasive microsensing system to simultaneously measure both $\mathrm{H}+$ and $\mathrm{O} 2$ fluxes around the pollen tube. Journal of Integrative Plant Biology 48(7), 823-831 (2006) 
3. Yin, L., Yu, S., Yue, X.: Scanning ion-selective electrode technique and the application in higher plant research. Progress in Natural Science 16(3), 262-266 (2006)

4. Richardson, D.C., Richardson, J.S.: Teaching molecular 3-D literacy. Biochemistry and Molecular Biology Education 30, 21-26 (2002)

5. Ding, Y., Xu, Y.: Non-invasive micro-test technology and its applications in biology and medicine. Physics 36(7), 548-558 (2007)

6. Rahman, M.F., Poo, A.-N.: An Application Oriented Test Procedure for Designing Microstepping Step Motor Controllers. IEEE Transactions On Industrial Electronics 35(4), 542-546 (1988)

7. Zhou, L., Yang, S., Gao, X.: Modeling of stepper motor control system and running curve simulation. Electric Machines And Control 15(1), 20-25 (2011)

8. Long, H., Zhai, C., Liu, X., et al.: Testing system of step motors and drives based on virtual instrument. Electric Machines And Control 10(6), 553-557 (2006)

9. Li, J., Gao, W.: Design of Step Motor Control System Based on LabVIEW. Science \& Technology Information 15, 85-86 (2010)

10. Jiang, J., Wu, B., Gao, J., et al.: Design of Stepper Motor Control by Virtual Instrumentation via Serial Interface. Electronic Science and Technology 25(8), 46-48 (2012)

11. Chen, D., Yao, C.: The design of PID control system based on LabVIEW for step motor. Industrial Instrumentation \& Automation 15, 48-49 (2005)

12. Chen, F., Li, J., Ma, S., et al.: Design of Step Motor Control System Using LabVIEW. Journal of Shanghai University (Natural Science Edition) 12(1), 89-92 (2006)

13. Chen, Y., Yang, C., Cao, J., et al.: Energy normalized photon-stimulated discharge spectra of polymer dielectric film. Electric Machines And Control 13(First Edition), 41-51 (2009)

14. Gu, J., Qin, S., Jin, L., et al.: Experiment Study On The Resonant Frequency Of Usm Based On LabVIEW. Proceedings of the CSEE 23(8), 91-95 (2003)

15. Xiang, X., Xia, P., Yang, S., et al.: Real-time Digital Simulation of Control System with LabVIEW Simulation Interface Toolkit. In: The 26th Chinese Control Conference, Hunan, pp. 318-322 (2007) 